\title{
Plataforma Web para Gestão dos Estágios Supervisionados nos Cursos de Graduação
}

\author{
Isaac B. S. de Souza ${ }^{1}$, Francisco das C. Rocha ${ }^{1}$, Rodrigo A. R. S. Baluz ${ }^{1}$ \\ ${ }^{1}$ Bacharelado em Ciência da Computação. \\ Universidade Estadual do Piauí (UESPI) - Parnaíba, PI - Brasil. \\ isaacwinfor@gmail.com, \{rocha, rodrigo.baluz\}@phb.uespi.br
}

\begin{abstract}
The supervised internship is a required element of many undergraduate courses serving as a way to connect the higher education student to the job market. This work aims to develop a web system that will systematize and automate the processes involving actors, students, teachers, course coordination and companies related to the academic activity of the supervised internship at the State University of Piaui. SIGESTAGIOS was available for testing by selected actors of the course under study for a period of 30 days, then evaluated for usability, achieving a good acceptance rate.
\end{abstract}

Resumo. O estágio supervisionado é um elemento obrigatório a muitos cursos de graduação servindo como forma de conectar o estudante do ensino superior ao mercado de trabalho. Este trabalho tem como objetivo apresentar um sistema web desenvolvido para sistematizar e automatizar os processos que envolvem os atores, alunos, professores, coordenação de curso e empresas relacionados à atividade acadêmica do estágio supervisionado no âmbito da Universidade Estadual do Piauí. O SIGESTAGIOS ficou disponível para testes por parte de atores selecionados do curso em estudo, durante um período de 30 dias, sendo em seguida, avaliado quanto sua usabilidade, alcançando um bom indice de aceitação.

\section{Introdução}

A tecnologia da informação (TI) vem auxiliando cada vez mais na tomada de decisões e proporcionando agilidade no andamento de processos da administração pública, fazendo com que os gestores possam ter maior controle sobre suas atribuições [Ferreira 2016]. Dentre as diferentes áreas que a TI vêm trazendo numerosos impactos, cita-se a educação [Oliveira e Moura 2016].

Nesse contexto, o curso de Ciência da Computação da Universidade Estadual do Piauí (UESPI) - Campus Parnaíba, sujeito de estudo deste trabalho, vem buscando melhorar as atividades educacionais com o auxílio da TI, servindo como modelo experimental para possível expansão aos demais campi da instituição.

Atualmente, o curso em estudo não dispõe de um sistema informatizado para controle de estágios supervisionados. Ou seja, todo o processo é feito de forma manual pelos atores envolvidos. Assim, tendo como base entrevistas realizadas com a coordenação de curso e de posse de documentos administrativos do estágio curricular, foi possível fazer uma abordagem mais criteriosa na definição dos requisitos funcionais para o desenvolvimento do sistema. 
O objetivo deste trabalho é sistematizar por meio de um software em plataforma web o processo de estágios supervisionados obrigatórios do curso de Bacharelado em Ciência da Computação da UESPI - Campus Parnaíba. Este sistema permitirá organizar e armazenar de forma centralizada os documentos referentes ao estágio, colaborando para um melhor controle e garantia da integridade dos dados, evitando assim, sobrecarregar os atores envolvidos com preenchimento de dados duplicados e manuseio de papel, além de disponibilizar uma biblioteca dinâmica de documentos e relatórios.

Para o desenvolvimento deste projeto foi criada uma aplicação web, aqui denominada SIGESTAGIOS, utilizando as ferramentas Django para back-end e Vue.js para o front-end. O front-end, que será escrito na forma de uma Single Page Application (SPA), acessará o back-end por meio de requisições utilizando o protocolo Hypertext Transfer Protocol (HTTP) assíncronas via Asynchronous JavaScript and XML (AJAX). Os dados serão disponibilizados e enviados de formulários para o back-end via uma Application Programming Interface (API) de arquitetura de software Representational State Transfer (REST), e persistidos em um banco de dados PostgreSQL. Haverá comunicação com serviços de terceiros, em particular com o serviço de dados do Instituto Brasileiro de Geografia e Estatística (IBGE), o que fornece uma API de localidades que será utilizada pela aplicação para exibir estados e cidades em campos de formulários de dados. Testes foram realizados com alunos do último período do curso de Bacharelado em Ciência da Computação e professores da instituição, além de supervisores das instituições concedentes de estágio. Por fim, foi aplicado um questionário para avaliação do nível de usabilidade do sistema.

Este trabalho está estruturado em cinco seções, sendo a primeira esta introdução. A segunda relaciona os trabalhos encontrados na literatura que usam de sistemas informatizados para melhorar a qualidade do serviço em cursos no ensino superior. A terceira aborda a metodologia bem como as ferramentas utilizadas para o desenvolvimento do protótipo. $\mathrm{Na}$ quarta seção são exibidas as principais telas do sistema e suas funcionalidades, como também são apresentados os resultados alcançados e suas análises. Por fim, são feitas as considerações finais e apresentação dos possíveis trabalhos futuros.

\section{Trabalhos Relacionados}

No trabalho de Silva et al. (2016), é proposto o desenvolvimento de um sistema de controle de Atividades Complementares (ACs) para melhorar as condições de gerenciamento de ACs, tanto para os estudantes quanto para o coordenador que avalia as atividades de todos os alunos. Os autores selecionaram e estudaram tecnologias tais como: Ruby On Rails como framework de aplicações web, MySQL como banco de dados e Git como ferramenta para controle de versão. Foram efetuadas reuniões durante o período letivo do curso de Bacharelado em Sistemas de Informação do Instituto Federal Fluminense para levantar requisitos do sistema e criar tarefas para um melhor entendimento sobre os módulos que compõem o mesmo. Segundo os autores, a maioria dos usuários percebeu uma melhoria no processo em relação ao método anterior.

No estudo de Almeida (2018), é apresentado que as metodologias atuais para controle do número de faltas na Universidade Federal de Ouro Preto (UFOP), não permitem a divulgação por parte dos docentes de maneira apropriada e quando um grande número de discentes são matriculados em determinada turma, o processo se 
torna custoso. Ainda, o autor propõe o desenvolvimento de um sistema web para informatizar e agilizar o processo, além de melhorar a metodologia de controle de assiduidade da UFOP. Durante o desenvolvimento utilizou técnicas de engenharia de software para auxílio em todo o processo. O autor desenvolveu o sistema utilizando a linguagem PHP e o framework Laravel em conjunto com o banco de dados MySql. Segundo ele, o sistema atende o processo de controle de faltas da UFOP e é capaz de substituir as metodologias utilizadas atualmente.

Leoncio (2018), por meio de pesquisas realizadas, constatou a ausência de um sistema para controle de Estágio no Departamento Acadêmico de Informática da Universidade Tecnológica Federal do Paraná (UTFPR), Campus Ponta Grossa, e que o armazenamento das informações é realizado com planilhas eletrônicas, tornando insegura $\mathrm{e}$ ineficiente a manipulação dos dados. $\mathrm{O}$ trabalho, apresenta $\mathrm{O}$ desenvolvimento de um sistema $w e b$, para controlar o histórico dos estágios obrigatórios e não obrigatórios, baseado em entrevistas com os usuários. Para o desenvolvimento da aplicação foi feita a modelagem do sistema através de diagramas da UML, a fim de abstrair as informações do mundo real. Segundo o autor, através da tecnologia empregada foi possível melhorar o desempenho das operações e a segurança dos dados.

Silva (2017), afirma que existe uma dificuldade pela maioria dos alunos da Faculdade de Computação da Universidade Federal de Uberlândia na fase de conclusão do curso por não encontrarem um tema adequado, e um orientador para $\mathrm{o}$ desenvolvimento do Trabalho de Conclusão de Curso (TCC). Diante disso, propôs o desenvolvimento de um sistema web, dedicado ao gerenciamento de TCCs, visando melhorar a comunicação entre alunos e professores, para gerar menos frustrações aos discentes e conflitos de informações. $\mathrm{O}$ autor frisa que, devido à alta usabilidade do sistema criado, os usuários não terão mais dificuldades na utilização das funcionalidades.

Dos trabalhos mencionados anteriormente, o do Leoncio (2018) é o que se assemelha ao trabalho aqui apresentado. Porém, os requisitos são bem diferentes, sendo eles baseados em documentos do processo de defesa de estágio da UTFPR, como mencionado pelo autor. Assim, o sistema foi construído com uma gama de funcionalidades que não constam nos trabalhos anteriores.

\section{Materiais e Métodos}

Esta seção descreve com detalhes as tecnologias e ferramentas de desenvolvimento utilizadas no projeto de construção do front-end e back-end da plataforma. Apresenta uma breve descrição do espaço utilizado como local de estudo para aplicação e testes da solução proposta. Os principais requisitos do sistema, assim como o diagrama de casos de uso e o diagrama do modelo lógico do banco de dados são apresentados. Detalhes sobre o processo de testes da aplicação, como quantidade de pessoas envolvidas e período de avaliação, foram descritos ao final desta seção.

\subsection{Ferramentas de desenvolvimento}

A escolha destas ferramentas se deu por critérios como produtividade; baixa curva de aprendizagem; documentação atualizada, bem escrita e de fácil e livre acesso; comunidades muito ativas e licença de uso. Em seguida serão listadas as principais ferramentas e/ou tecnologias utilizadas no desenvolvimento do sistema. 
- Back-End

o Framework Django

○ Django REST Framework

○ SGBD PostgreSQL

- Front-End

○ Framework Vue.js

o Framework Vuetify

As subseções a seguir referem-se a uma descrição sucinta de cada uma das tecnologias citadas anteriormente.

\subsubsection{Framework Django}

Django é um framework para desenvolvimento web de alto nível, escrito na linguagem de programação Python, que incentiva o rápido desenvolvimento de aplicações com design limpo e prático. Criado por desenvolvedores experientes, para que se possa escrever aplicações web sem necessidade de "reinventar a roda". É uma ferramenta gratuita e open-source [Django 2005]. Para o desenvolvimento do software deste trabalho, foi utilizada a versão 1.11 do framework.

\subsubsection{Django REST Framework}

O Django REST Framework é uma biblioteca Python que permite a criação de APIs REST da web de forma simples e flexível em projetos Django, que proporciona produtividade [Brendon 2016]. Este trabalho fez uso da versão 3.9 do Django REST Framework.

\subsubsection{SGBD PostgreSQL}

O PostgreSQL é um poderoso Sistema Gerenciador de Banco de Dados (SGBD) objetorelacional open-source, que usa a linguagem SQL para manipulação dos dados [Postgresql 2019]. Tem como função principal, armazenar dados de forma segura, se apoiando a boas práticas. Pode ser utilizado tanto em aplicações de pequeno porte quanto em aplicações de grande porte voltadas para a internet, onde vários usuários utilizarão de forma simultânea [Dionisio 2015]. Este trabalho fez uso da versão 11.0 do PostgreSQL.

\subsubsection{Framework Vue.js}

O Vue.js é um framework progressivo para a construção de interfaces de usuário, escrito em JavaScript. Ele faz parte da camada visual (view layer) do modelo de arquitetura MVC, sendo fácil de integrar com outras bibliotecas existentes. Ele é distribuído sob a licença MIT e também pode ser usado na criação de sofisticadas Single Page Applications [Vue 2014], como no caso deste trabalho, onde foi usada a versão 2.5 do framework. 


\subsubsection{Framework Vuetify}

Vuetify é um framework para criar belas aplicações com interface responsiva, escrito em Vue.js, possui uma boa quantidade de componentes reutilizáveis, uma documentação de fácil acesso, disponibilizado sob a licença MIT e é baseado no Material Design [Vuetify 2016]. O Material Design é uma metodologia de design com foco nas melhores práticas para projetos de interface de usuário, desenvolvido pela Google [Rallo 2017]. Neste trabalho foi utilizada a versão 1.4 do Vuetify.

\subsection{Local de Estudo}

O Campus Professor Alexandre Alves de Oliveira da Universidade Estadual do Piauí (UESPI) com sede em Parnaíba-PI foi criado em 1991. Atualmente oferta os seguintes cursos de graduação: Bacharelado em Direito; Bacharelado em Ciência da Computação; Bacharelado em Enfermagem; Bacharelado em Odontologia; Bacharelado em Engenharia Agronômica; Licenciatura em Letras-Inglês; Licenciatura em Ciências Biológicas; Licenciatura em História; Licenciatura em Letras-Português; Licenciatura em Pedagogia; Licenciatura em Ciências Sociais e Licenciatura em Filosofia. A instituição possui como principais objetivos ser referência em ensino superior e oferecer um serviço educacional de qualidade para toda a sociedade, e não apenas parnaibana.

\subsection{Requisitos do Sistema}

Para chegar à definição dos requisitos necessários para o desenvolvimento da proposta foram realizadas reuniões junto à coordenação do curso de Ciência da Computação da UESPI-Parnaíba, assim como a análise de documentos referentes ao estágio supervisionado. Diante disso, a listagem abaixo exibe resumidamente os 10 principais requisitos que caracterizam o cenário aqui proposto.

1. O coordenador de curso insere no sistema os alunos aptos a fazerem estágio.

2. Os alunos recebem um e-mail e a partir daí entram no sistema e preenchem a ficha de inscrição do estágio.

3. O coordenador de curso pode imprimir um relatório chamado "Mapa de Estágio".

4. Na ficha de inscrição o aluno pode, caso necessário, cadastrar uma instituição que não possui convênio com a UESPI.

5. A coordenação imprime o termo de compromisso e, solicita se necessário, a confirmação de contrato de convênio entre as instituições.

6. O aluno e seu orientador devem preencher o "Plano de Estágio", que deverá ser validado pelo supervisor de estágio da empresa/instituição.

7. O aluno deverá a cada dia de estágio fazer o registro de sua permanência na empresa, que deve ser validado pelo orientador e supervisor da empresa/instituição.

8. A cada mês de estágio o aluno preenche a "Ficha de Acompanhamento", que também deve ser validado pelo orientador e supervisor.

9. Ao final, o aluno deve preencher o "Relatório Final" de estágio, que deve ser validado pelos mesmos envolvidos. 
10. O orientador e supervisor, ao final do ciclo, devem preencher a "Ficha de Desempenho", avaliando o aluno estagiário.

\subsection{Modelos do Sistema}

Após o processo de levantamento de requisitos junto a coordenação do curso de Ciência da Computação da UESPI em Parnaíba, foram criados diagramas para a representação das funcionalidades requeridas na gestão de estágios supervisionados, utilizando para tal a Linguagem de Modelagem Unificada (Unified Modeling Language - UML). A UML é uma linguagem para a modelagem de sistemas de computador, que oferece uma forma gráfica para a visualização dos requisitos do sistema, baseada no paradigma da orientação a objetos [Booch, Rumbaugh e Jacobson 2005; Freitas 2008].

A fase de modelagem do sistema se deu primeiramente por meio da criação do diagrama de casos de uso. Os casos de uso demonstram uma visão estática das principais funcionalidades e interações dessas funcionalidades com os usuários do sistema sem a necessidade de detalhes técnicos de como o sistema faz [Booch, Rumbaugh e Jacobson 2005; Ribeiro 2012].

A Figura 1 apresenta as funcionalidades, visíveis aos atores, que serão fornecidas pelo sistema, representadas por casos de uso e os respectivos usuários que poderão executá-las, representados por atores. Neste diagrama estão listados apenas os casos de uso com as principais funções do sistema. É importante mencionar que antes de tudo, todos os usuários precisarão fornecer suas credenciais de acesso (e-mail $\mathrm{e}$ senha) para executar as ações dentro do sistema. Neste diagrama não foram representadas as funcionalidades de login e logout, assim como as operações básicas de CRUD no banco de dados.

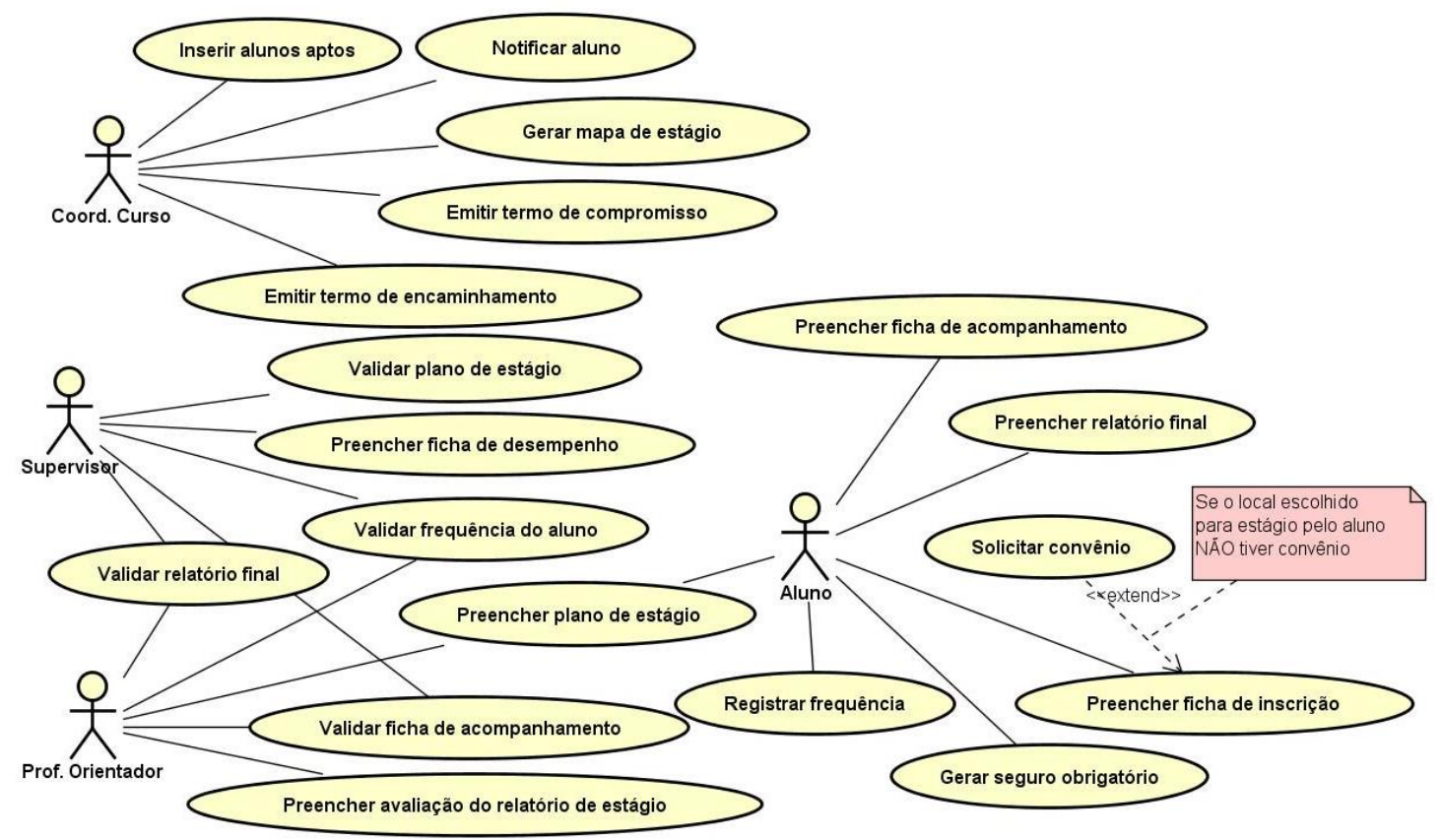

Figura 1. Diagrama de Casos de Uso simplificado.

De acordo com o diagrama de casos de uso, existem diferentes usuários do sistema: Coordenador de Curso, Supervisor de Estágio, Professor Orientador e Aluno. 
No caso de uso "Inserir alunos aptos" o coordenador irá cadastrar os alunos aptos a fazerem estágio e poderá imediatamente notificá-los através do caso de uso "Notificar aluno". Esta notificação realizada de forma manual pelo coordenador se faz importante para lhe permitir um controle quanto aos alunos já notificados, que ainda realizaram o preenchimento na plataforma dos documentos iniciais necessários para o início do estágio supervisionado.

No caso de uso "Preencher ficha de inscrição" o aluno poderá, caso o local escolhido para estágio não possua convênio com a instituição, executar o caso de uso "Solicitar convênio". Nesse caso de uso, o discente preencherá um formulário com os dados referentes à empresa concedente, necessário para efetivação do Termo de Convênio institucional entre a IES e a empresa. Após o envio desse formulário, o coordenador de curso será notificado automaticamente sobre a necessidade de solicitar convênio institucional. A partir deste momento, o coordenador ficará responsável pela conclusão do convênio junto ao setor competente da instituição.

Após a definição dos diagramas UML foi criado o modelo lógico do banco de dados. O modelo lógico é uma representação de um banco de dados em um nível de abstração compreendido pelo usuário do SGBD [Heuser 2009]. A Figura 2 mostra o diagrama do modelo lógico do banco de dados.

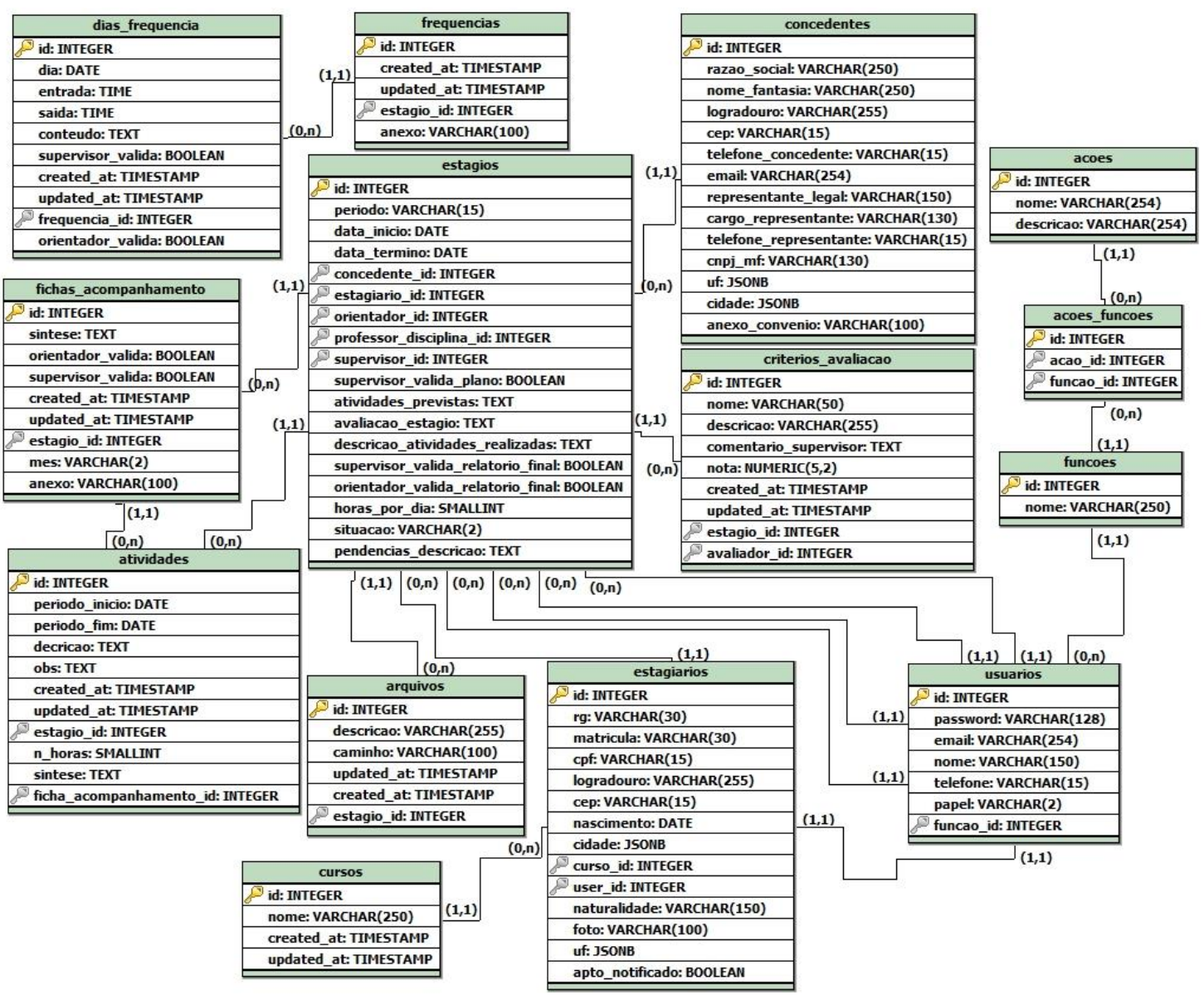

Figura 2. Modelo lógico do banco de dados.

Ao se inserir um aluno apto, a tabela "estagiarios" somente será preenchida quando da efetivação da inscrição por parte do discente. A tabela "criterios_avaliacao" 
terá valores padrões quando os discentes forem efetivando suas inscrições na plataforma. Esta tabela armazenará tanto os critérios de avaliação do desempenho do aluno no estágio quanto os de avaliação do relatório final de estágio. A tabela "arquivos" armazenará informações dos anexos de cada estágio como, a descrição do anexo (Plano de Estágio, Relatório Final etc.) e o caminho, que é o local onde se encontrará $\mathrm{o}$ anexo no servidor.

\subsection{Teste do Sistema}

Para testar a plataforma SIGESTAGIOS, foram convidados 13 voluntários, sendo estes com perfis de atores que se relacionam com o estágio supervisionado no ensino superior: 1 coordenador de curso, 7 alunos de graduação, 2 professores orientadores e 3 supervisores ligados às empresas concedentes do estágio. O sistema foi implantado no dia 04 de junho de 2019, data também de início dos testes. O usuário do tipo coordenador de curso foi cadastrado no sistema. Este usuário é o principal administrador do sistema e quem dá início ao processo para que os outros possam ter acesso. Os convidados tiveram um breve treinamento e foram cadastrados no sistema. Também foi disponibilizado um documento para cada usuário, descrevendo o passo a passo de execução das tarefas dentro do sistema. O período de testes terminou no dia 04 de julho de 2019.

\section{Análise e Discussão dos Resultados}

Nesta seção serão apresentadas as principais telas do sistema com suas funcionalidades e, por conseguinte, os resultados dos testes efetuados por meio do questionário SUS. É importante mencionar que os dados apresentados nas figuras de telas do sistema são somente ilustrativos.

\subsection{A plataforma SIGESTAGIOS}

Na página de perfil (Figura 3), primeira tela do sistema a ser exibida ao usuário após ter sido identificado na tela de login, pode-se observar ao lado esquerdo da tela o menu de funcionalidades que, dependerá da função (Aluno, Coordenador de curso, Orientador ou Supervisor) do usuário que fez o login.

Na Figura 3, pode ser observado o painel de perfil sob a ótica de um coordenador de curso com as opções de alterar senha e gerar relatórios específicos deste usuário. As opções do menu para este usuário são: "Meu Perfil", "Alunos Aptos", "Matriculados", "Orientandos", "Concedentes", "Usuários" e "Sair". Clicando em "Alunos Aptos" será exibida a tela de listagem de todos os alunos aptos a fazerem estágio.

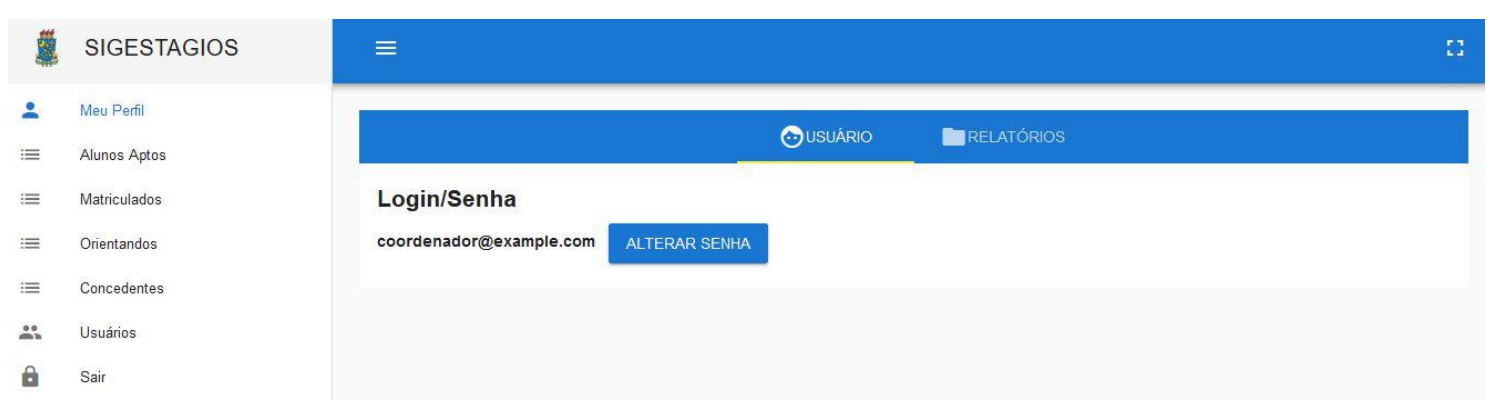

Figura 3. Tela de perfil de usuário coordenador de curso. 
$\mathrm{Na}$ tela de alunos aptos (Figura 4), o coordenador de curso terá as seguintes opções: "ENVIAR E-MAIL" para notificar ao aluno que ele deverá preencher suas informações de estágio, ou seja, é um processo manual, com isso o coordenador poderá notificá-lo novamente a partir da ocorrência de um problema como, por exemplo, o cadastramento de um endereço de e-mail equivocado; "NOVO ALUNO" para adicionar aluno apto; um campo de texto intitulado "Pesquisar" que poderá ser usado para buscar alunos ou pelo nome ou pela matrícula; e um botão com um ícone de lápis que permitirá a alteração dos dados de aluno apto. Além dos botões de ordenação no cabeçalho da tabela e dos botões de paginação vistos abaixo dela. Na medida em que os alunos vão sendo notificados para a realização da inscrição no estágio, o botão "ENVIAR EMAIL" ficará na cor verde, representando exatamente a confirmação da notificação.

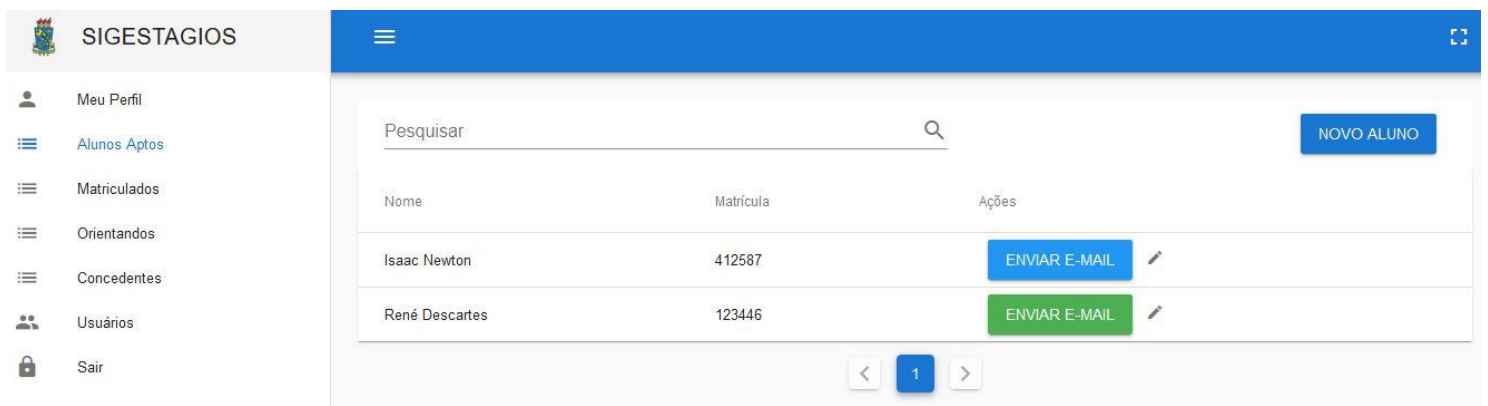

Figura 4. Tela de listagem de alunos aptos.

O coordenador de curso também pode ser orientador. Na fase de implementação caso o usuário logado fosse do tipo coordenador de curso, optou-se por dividir a listagem dos estudantes em duas páginas distintas. A página dos matriculados, que exibirá todos os alunos que já completaram o cadastro de seus estágios e a dos orientandos, que exibirá os alunos que escolheram o coordenador de curso inclusive como orientador. A Figura 5 mostra a página de alunos matriculados, que apresenta as mesmas opções de busca, ordenação e paginação já mencionados para os alunos aptos, além de algumas outras opções de ações. Os botões da cor vermelha na coluna "Ações" significam que serão gerados documentos PDFs de forma dinâmica com os dados dos respectivos estagiários.

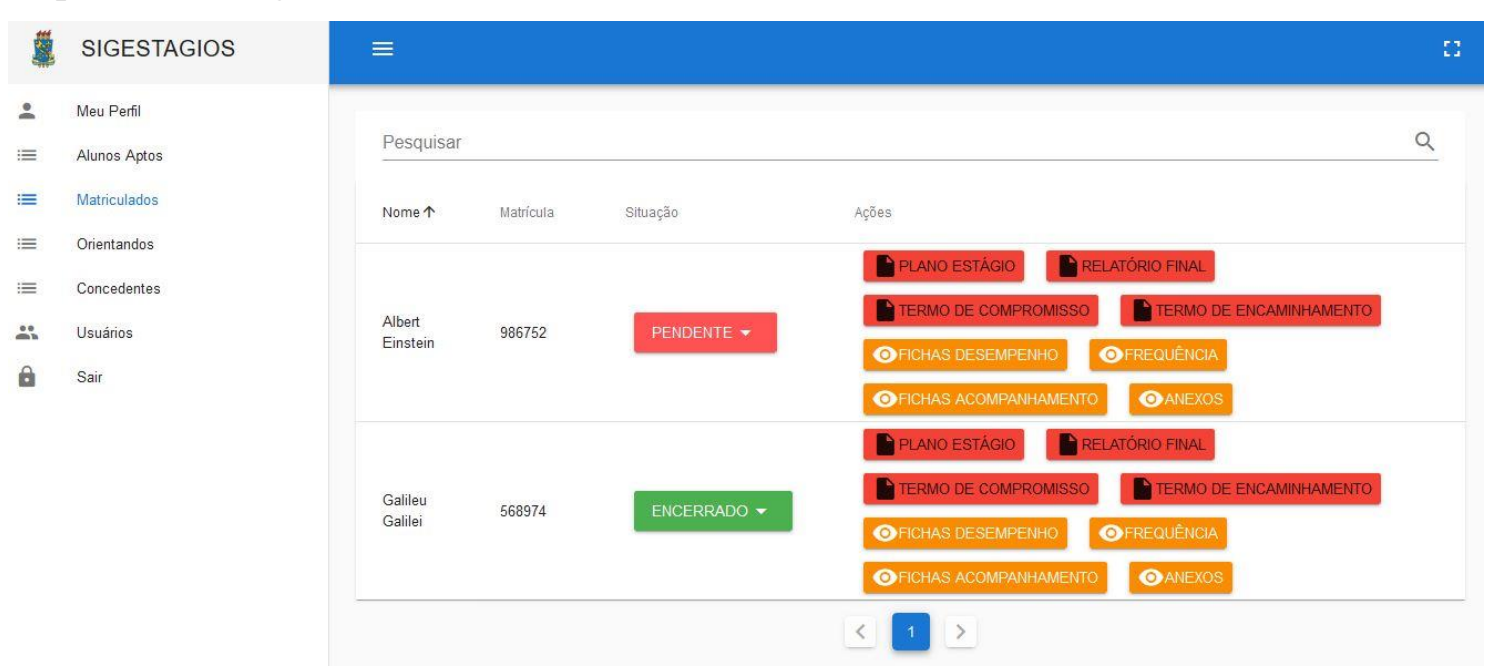

Figura 5. Tela de listagem de alunos matriculados. 
$\mathrm{Na}$ página de perfil do estagiário, mais especificamente na aba de frequência (Figura 6), o aluno estagiário poderá adicionar os dias da frequência de acordo com o mês selecionado no campo "Mês". Ao final ele terá a opção de notificar os atores interessados (professor orientador e supervisor de estágio) clicando no botão "NOTIFICAR INTERESSADOS". Clicando no botão "FREQUÊNCIA", será gerado um documento PDF com os dados do modelo referentes ao mês selecionado.

Após concluir a frequência para todos os meses em que ele estagiou, o aluno precisará anexar os documentos assinados no sistema. Na aba "ANEXOS" ele fará o upload desses documentos, mas antes terá que uni-los em um único arquivo.

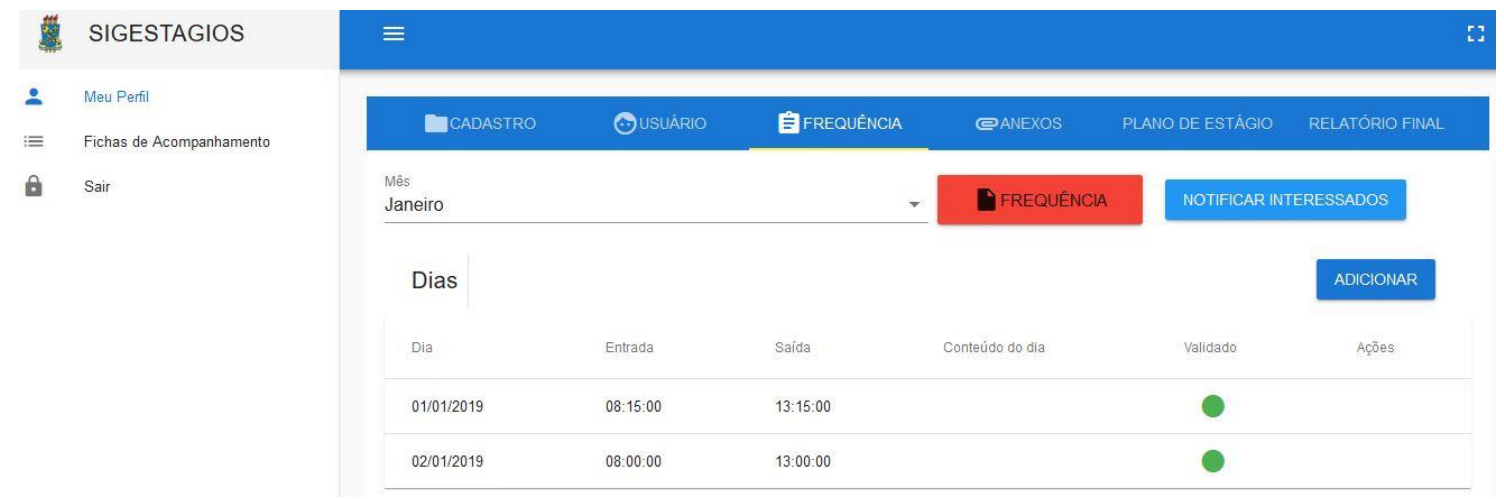

Figura 6. Tela de frequência do aluno estagiário.

Na página de listagem de supervisionados, assim como na página de listagem de orientandos, terá o botão nomeado "DESEMPENHO". Nesta página, ao clicar no botão será exibida tanto ao supervisor de estágio quanto ao orientador uma janela com um formulário para preenchimento da ficha de avaliação de desempenho (no caso do orientador, a ficha de avaliação de desempenho do relatório final) do respectivo estagiário, como é apresentado no exemplo da Figura 7.

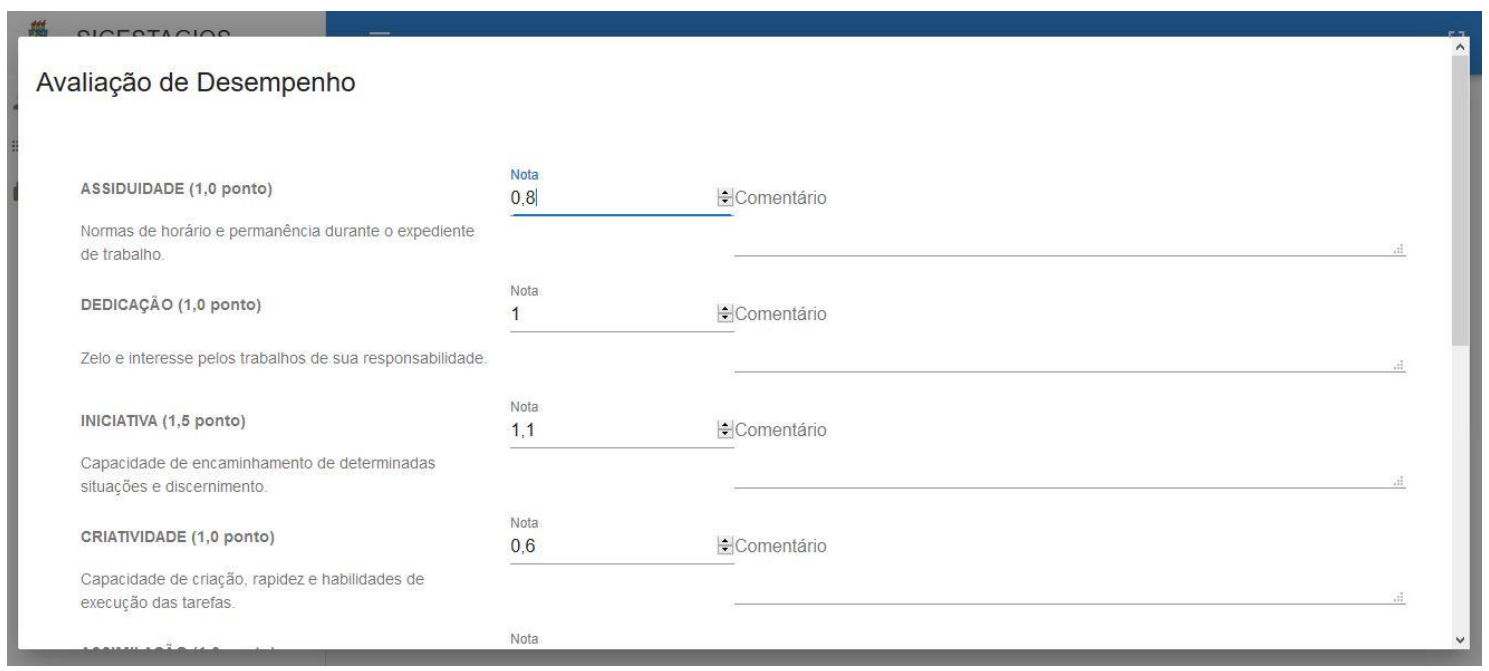

Figura 7. Tela com formulário de avaliação de desempenho do estagiário.

\subsection{Avaliação de Usabilidade}

A Escala de Usabilidade do Sistema - System Usability Scale (SUS) é um método simples, porém de fácil avaliação do problema de usabilidade de um sistema, criado em 
1986 por John Brooke. Ele pode ser utilizado para avaliar qualquer tipo de interface, produtos, serviços, hardware, software, websites, aplicações [Teixeira 2015; Sauro 2011].

Para avaliação da usabilidade do SIGESTAGIOS, foi disponibilizado um questionário com as 10 primeiras perguntas apoiadas no SUS, adaptadas de Sauro (2011) por Teixeira (2015), e aqui acrescentado de outras 2 questões, que permitem avaliar o nível funcional de sua aplicação aos cursos de graduação no ensino superior. A seguir, serão listadas as 12 perguntas básicas do questionário que foi aplicado em um formulário online, após a finalização dos testes dos usuários.

1. Eu acho que gostaria de usar esse sistema com frequência.

2. Eu acho o sistema desnecessariamente complexo.

3. Eu achei o sistema fácil de usar.

4. Eu acho que precisaria de ajuda de uma pessoa com conhecimentos técnicos para usar o sistema.

5. Eu acho que as várias funções do sistema estão muito bem integradas.

6. Eu acho que o sistema apresenta muita inconsistência.

7. Eu imagino que as pessoas aprenderão como usar esse sistema rapidamente.

8. Eu achei o sistema atrapalhado de usar.

9. Eu me senti confiante ao usar o sistema.

10. Eu precisei aprender várias coisas novas antes de conseguir usar o sistema.

11. Eu usaria o sistema caso fosse implantado no curso.

12. Eu acredito que o sistema pode contribuir com a gestão dos estágios supervisionados em cursos de graduação.

Cada questão assume um formato de resposta [Sauro 2011], como demonstrado na Figura 8. Onde a escala, fundamentada na escala Likert, varia de 1 a 5, com 1 significando "Discordo Completamente" e 5 significando "Concordo Completamente" [Teixeira 2015]. Likert (1932) desenvolveu esta escala para medir até que ponto as pessoas concordam ou discordam de uma série de afirmações sobre um tópico. Aos entrevistados podem ser apresentadas cinco a sete ou até nove opções de respostas précodificadas, sendo uma como ponto neutro [Mcleod 2008].

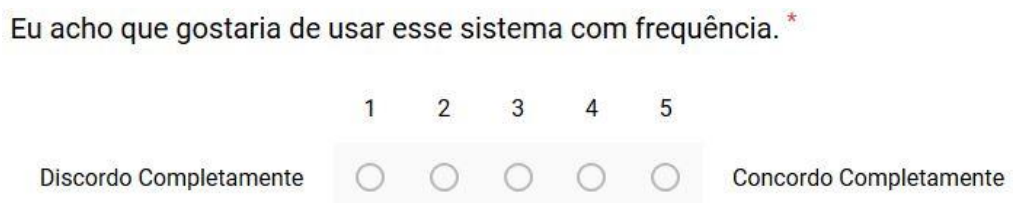

Figura 8. Formato de resposta de acordo com a metodologia do SUS.

O principal objetivo deste questionário foi a coleta de dados da usabilidade do sistema no contexto do estágio supervisionado do curso de Computação da UESPIParnaíba. Ele foi criado e disponibilizado online através do aplicativo Formulários Google. Na aquisição das respostas não foi solicitado nenhum tipo de identificação dos voluntários. 
O questionário SUS foi uma maneira quantitativa de medir a satisfação dos usuários na realização das tarefas desempenhadas pelo sistema. O Formulários Google, foi utilizado para recuperar as respostas dos usuários e, em seguida, o Planilhas Google, utilizado para manipular os cálculos e obter o score.

Pode-se observar através da Tabela 1 as respostas obtidas no questionário. Foram 13 voluntários como mencionado na seção 3. No cabeçalho da tabela nota-se que na primeira coluna, para cada linha representa-se um voluntário. A expressão "Q1" na segunda coluna significa "Questão 1", e assim sucessivamente para as demais colunas. As questões Q11 e Q12 não foram utilizadas para fins de cálculos do score final, respeitando o modelo original do questionário SUS, que avalia usabilidade. As questões foram adicionadas pelo autor, como forma de validação funcional da plataforma. Conforme observado nas colunas Q11 e Q12 da Tabela 1, observamos que 92,31\% dos participantes manifestaram que usariam a aplicação caso seja implantada pelo curso na IES. Também, se mostraram favorável quanto da importância da ferramenta em contribuir com a gestão dos estágios supervisionados em cursos de graduação.

Tabela 1. Respostas obtidas no questionário.

\begin{tabular}{ccccccccccccc}
\hline Voluntário & Q1 & Q2 & Q3 & Q4 & Q5 & Q6 & Q7 & Q8 & Q9 & Q10 & Q11 & Q12 \\
\hline 1 & 5 & 1 & 5 & 2 & 5 & 2 & 5 & 1 & 5 & 1 & 5 & 5 \\
2 & 4 & 2 & 5 & 1 & 4 & 3 & 4 & 2 & 4 & 1 & 5 & 5 \\
3 & 4 & 1 & 5 & 1 & 4 & 2 & 5 & 2 & 5 & 1 & 5 & 5 \\
4 & 5 & 2 & 3 & 2 & 4 & 2 & 5 & 1 & 4 & 1 & 5 & 5 \\
5 & 5 & 1 & 5 & 1 & 5 & 1 & 4 & 1 & 5 & 1 & 5 & 5 \\
6 & 5 & 3 & 4 & 2 & 3 & 3 & 5 & 1 & 4 & 1 & 5 & 5 \\
7 & 5 & 1 & 4 & 1 & 5 & 1 & 5 & 1 & 5 & 1 & 5 & 5 \\
8 & 5 & 1 & 5 & 3 & 5 & 1 & 4 & 1 & 5 & 1 & 5 & 5 \\
9 & 3 & 4 & 2 & 4 & 3 & 3 & 3 & 3 & 3 & 3 & 5 & 5 \\
10 & 3 & 1 & 4 & 1 & 3 & 2 & 5 & 1 & 5 & 1 & 5 & 5 \\
11 & 5 & 1 & 5 & 1 & 4 & 2 & 5 & 1 & 5 & 1 & 5 & 5 \\
12 & 5 & 1 & 5 & 1 & 4 & 1 & 5 & 1 & 5 & 1 & 5 & 5 \\
13 & 5 & 5 & 2 & 5 & 3 & 5 & 2 & 5 & 2 & 1 & 3 & 3 \\
\hline
\end{tabular}

O score final é obtido através dos passos a seguir, definidos por Sauro (2011):

1. Para questões ímpares, subtraia 1 do valor da resposta do usuário.

2. Para questões pares, subtraia o valor da resposta de usuário de 5 .

3. Então, some todos os valores convertidos das dez questões para cada usuário, e multiplique por 2,5. 
O score final pode variar de 0 a 100 e é atingido pelo cálculo da média dos resultados de cada voluntário, que no caso deste trabalho foi de aproximadamente 81,92. De acordo com Sauro (2011), a média do score para uma boa avaliação é de 68 pontos. Esse índice de referência foi adotado pela comunidade científica devido a ampla aplicabilidade do questionário em diversos estudos. Sendo assim, é possível observar que o resultado adquirido está bem acima da média.

\section{Considerações Finais}

São muitas as dificuldades apresentadas na gestão do estágio curricular supervisionado. No entanto, com o auxílio das Tecnologias da Informação e Comunicação é possível oportunizar novas experiências aos estudantes em campo de estágio, assim como permitir aos gestores do ensino superior e empresas/instituições concedentes uma forma dinâmica e automatizada no controle de documentos e processos. Diante desse cenário, a plataforma SIGESTAGIOS foi projetada a fim de mitigar possíveis erros e redundância no preenchimento dos dados e facilitar a geração dos relatórios referentes ao estágio.

Este projeto teve como objetivo fundamental entregar uma ferramenta tecnológica que possibilitasse um controle centralizado e facilitado dos documentos, tanto por parte dos estudantes como por parte dos docentes e supervisores do componente de estágio curricular da UESPI-Parnaíba. Não há mais necessidade de impressões desnecessárias destes documentos, visto que eles são mantidos na base de dados do sistema, ou por meio de preenchimento de formulários ou por meio de upload de arquivos. Além de ser possível saber se o documento foi validado e/ou revisado pelo docente e/ou pelo supervisor. É importante mencionar, que, não era objetivo deste trabalho desenvolver uma interface de programação para integração com o sistema acadêmico já existente da IES. Porém, o sistema atende à rotina geral de comunicação em relação aos critérios de execução de um Estágio no Ensino Superior. Podendo ser perfeitamente usado em qualquer IES.

Para conseguir atingir os objetivos foi fundamental o estudo e uso de técnicas de desenvolvimento de software e de tecnologias como o framework Django, Django REST Framework, Vue.js, assim como integração com a API do IBGE na aplicação.

De acordo com o resultado do questionário de usabilidade SUS, o sistema obteve um score bem acima da média. Isso quer dizer que o sistema foi, de maneira geral, bem intuitivo para os usuários voluntários. Além de apresentar uma boa usabilidade, o desenvolvimento dessa ferramenta possibilita um passo inicial quanto a futuros trabalhos que tenham como objetivo o melhoramento da plataforma. Outra contribuição é o próprio sistema como ferramenta que pode auxiliar o corpo docente e discente da instituição ao qual foi planejado e desenvolvido e possivelmente outras instituições.

Como trabalhos futuros, no que se refere a este sistema, além da adição de funcionalidades, como o desenvolvimento de uma API para integração com o sistema acadêmico já existente na IES, o gerenciamento dos documentos sem a necessidade de upload de arquivos, é importante ressaltar não só o aperfeiçoamento de algumas das funcionalidades, como, por exemplo, as mensagens de notificação aos atores envolvidos no processo, mas também a realização de mais testes para validação da proposta. 


\section{Referências}

Almeida, B. C. (2018). Desenvolvimento de um Sistema Web para Controle de Faltas da Universidade Federal de Ouro Preto. Universidade Federal de Ouro Preto. Monografia.

Booch, G., Rumbaugh, J., e Jacobson, I. (2005). UML: Guia do Usuário. Elsevier Brasil.

Brendon, H. (2016). Criando API REST com Django Rest Framework 1/10. Grupy RN. Disponível em: <https://medium.com/grupy-rn/criando-api-rest-com-django-restframework-1-10-e77208f7e5b9>. Acesso em: 19 jul. 2019.

Dionisio, E. J. (2015). PostgreSQL Tutorial. DevMedia. Disponível em: $<$ https://www.devmedia.com.br/postgresql-tutorial/33025>. Acesso em: 18 jul. 2019.

Django. (2005). Django makes it easier to build better Web apps more quickly and with less code. Página inicial. Disponível em: $<$ https://www.djangoproject.com/>. Acesso em: 10 dez. 2018.

Ferreira, J. C. (2016). A tecnologia como instrumento na gestão pública: um estudo de caso do processo administrativo digital no Tribunal Regional Eleitoral do Maranhão. Universidade Federal do Maranhão. Monografia.

Freitas, M. L. (2008). UML. DevMedia. Disponível em: $<$ https://www.devmedia.com.br/uml/8579>. Acesso em: 18 jul. 2019.

Heuser, C. A. (2009). Projeto de banco de dados: Volume 4 da Série Livros didáticos informática UFRGS. Bookman Editora.

Leoncio, D. (2018). Sistema para controle de estágio supervisionado do departamento acadêmico de informática. Universidade Federal do Paraná. Monografia.

Mcleod, S. (2008). Likert Scale. SimplyPsychology. Disponível em: $<$ https://www.simplypsychology.org/likert-scale.html >. Acesso em: 23 jul. 2019.

Nascimento, E. S. (2017). Modelagem e Protótipo de um Sistema para Gerenciamento de Reuniões. Instituto Federal de Educação, Ciência e Tecnologia de Minas Gerais Campus Formiga. Monografia.

Oliveira, C., Moura, S. P. e Sousa, E. R. (2015). TIC'S na educação: a utilização das tecnologias da informação e comunicação na aprendizagem do aluno. PEDAGODIA EM AÇÃO, v. 7, n. 1, p. 75-95.

Postgresql. (1996). About PostgreSQL. Disponível em: $<$ https://www.postgresql.org/about/>. Acesso em: 08 jun. 2019.

Rallo, R. (2019). Material Design: aprenda tudo sobre o design do Google!. rockcontent. Disponível em: <https://rockcontent.com/blog/material-design/>. Acesso em: 23 jul. 2019.

Ribeiro, L. (2012). O que é UML e Diagramas de Caso de Uso: Introdução Prática à UML. DevMedia. Disponível em: <https://www.devmedia.com.br/o-que-e-uml-ediagramas-de-caso-de-uso-introducao-pratica-a-uml/23408 $>$. Acesso em: 09 jun. 2019. 
Sauro, J. (2011). Measuring usability with the system usability scale (SUS). MeasuringU. Disponível em: <https://measuringu.com/sus/>. Acesso em: 19 jul. 2019.

Silva, A. S. S., Conceição, E. B. e Silva, L. S. (2016). Desenvolvimento de um Sistema de Controle de Atividades Complementares em Ruby On Rails - Um Estudo das Gems Bootstrap, Devise e Prawn. Instituto Federal Fluminense. Monografia.

Silva, M. R. R. (2017). Projeto e Desenvolvimento de um Sistema para Gerenciamento de Trabalhos de Conclusão de Curso. Universidade Federal de Uberlândia. Monografia.

Teixeira, F. (2015). O que é o SUS (System Usability Scale) e como usá-lo em seu site. UX Collective BR. Disponível em: $<$ https://brasil.uxdesign.cc/o-que-e-o-sus-systemusability-scale-e-como-usa-lo-em-seu-site-6d63224481c8>. Acesso em: $10 \mathrm{dez}$. 2018 .

Vue. (2014). The Progressive JavaScript Framework. Página inicial. Disponível em: $<$ https://vuejs.org/>. Acesso em: 23 jul. 2019.

Vuetify. (2016). Material Design Component Framework. Página inicial. Disponível em: $<$ https://vuetifyjs.com/en/>. Acesso em: 23 jul. 2019. 